\title{
Gut microbiota and their metabolites in the progression of non-alcoholic fatty liver disease
}

\author{
Jin Zhou', Madhulika Tripathi', Rohit A. Sinha², Brijesh Kumar Singh', Paul M. Yen ${ }^{1,3,4}$ \\ 1Program of Cardiovascular and Metabolic Disorders, Duke-NUS Medical School, Singapore 169857, Singapore. \\ 2Department of Endocrinology, Sanjay Gandhi Postgraduate Institute of Medical Sciences, Lucknow 226014, India. \\ ${ }^{3}$ Duke Molecular Physiology Institute, Durham, NC 27701, USA. \\ ${ }^{4}$ Duke University School of Medicine, Durham, NC 27710, USA.
}

Correspondence to: Dr. Jin Zhou, Program of Cardiovascular \& Metabolic Disorders, Duke-NUS Medical School, 8 College Road, Singapore 169857, Singapore. E-mail: jin.zhou@duke-nus.edu.sg

How to cite this article: Zhou J, Tripathi M, Sinha RA, Singh BK, Yen PM. Gut microbiota and their metabolites in the progression of non-alcoholic fatty liver disease. Hepatoma Res 2021;7:11x. http://dx.doi.org/10.20517/2394-5079.2020.134

Received: 24 Oct 2020 First Decision: 1 Dec 2020 Revised: 21 Dec 2020 Accepted: 29 Dec 2020 Published: 13 Jan 2021

Academic Editor: Mark Alan Feitelson Copy Editor: Miao Zhang Production Editor: Jing Yu

\begin{abstract}
Non-alcoholic fatty liver disease (NAFLD) is the most prevalent liver disorder worldwide. It comprises a spectrum of conditions that range from steatosis to non-alcoholic steatohepatitis, with progression to cirrhosis and hepatocellular carcinoma. Currently, there is no FDA-approved pharmacological treatment for NAFLD. The pathogenesis of NAFLD involves genetic and environmental/host factors, including those that cause changes in intestinal microbiota and their metabolites. In this review, we discuss recent findings on the relationship(s) of microbiota signature with severity of NAFLD and the role(s) microbial metabolites in NAFLD progression. We discuss how metabolites may affect NAFLD progression and their potential to serve as biomarkers for NAFLD diagnosis or therapeutic targets for disease management.
\end{abstract}

Keywords: Non-alcoholic fatty liver disease, gut microbiome, gut microbiota metabolites

\section{INTRODUCTION}

Non-alcoholic fatty liver disease (NAFLD) is one of the most prevalent chronic liver diseases worldwide ${ }^{[1]}$. NAFLD is characterized by fat accumulation and defined by the presence of steatosis in $>5 \%$ of hepatocytes according to histological analysis without any history of significant alcohol consumption or viral hepatitis ${ }^{[2]}$. NAFLD includes two pathological conditions: non-alcoholic fatty liver (NAFL) and 
non-alcoholic steatohepatitis (NASH). Among the two conditions, NASH represents a more severe form and is characterized by progressive inflammation, hepatocyte death, and fibrosis ${ }^{[2]}$. Recently, it has been proposed that NAFLD be renamed as metabolic dysfunction associated with fatty liver disease (MAFLD) to highlight its relationship to metabolic conditions such as obesity, diabetes, hypertriglyceridemia, hypercholesterolemia, and atherosclerosis. If universally adopted, this new nomenclature and definition will have a great impact on clinical practice ${ }^{[3,4]}$, particularly with respect to the diagnosis of patients and endpoints for clinical trials ${ }^{[5]}$. However, in alignment with previously published literature, the current review continues to use the term "NAFLD".

NAFLD is a chronic and complex disease that may be attributed to a combination of genetic and environmental factors. NAFLD is highly associated with obesity, type 2 diabetes mellitus (T2DM), and dyslipidemia ${ }^{[1]}$. "Multiple parallel hits" have been postulated to explain the complex molecular pathogenesis underlying the evolution from NAFLD to $\mathrm{NASH}^{[6]}$. Alteration of gut microbiota is thought to be one of the hits that contributes to pathogenesis of NAFLD. Indeed, the gut microbiome affects the lipid metabolism, apoptosis, inflammation, and fibrosis during NAFLD progression. Therefore, the aim of this review is to highlight the molecular mechanism(s) for microbiota induction of NAFLD progression and the current strategies to manage NAFLD by manipulating microbiota or its metabolites.

\section{GUT MICROBIOTA IN NAFLD PROGRESSION}

The intestine harbors a large quantity of microorganisms, mostly comprising of bacteria, that are collectively called gut microbiota. Their total number in the body is estimated to be approximately 40 trillion, which is close to the total number of human cells in a person, and their total mass is approximately $0.2 \mathrm{~kg}^{[7]}$. Bacteria belong to specific taxonomic groups, comprising phyla, classes, orders, families, genera, and species. More than $90 \%$ of the microbiota in the gut microbiome belong to two phyla, Firmicutes and Bacteroidetes ${ }^{[8]}$. Recent advances in molecular biology techniques, particularly in sequencing and bioinformatic analysis, enable detailed characterization of the composition and diversity of the gut microbiome. Microbial diversity begins within the first few hours after birth and is shaped during childhood and adolescence when the diet becomes more diverse and the immune system matures. In contrast, the composition of adult microbiota in the gut remains relatively stable ${ }^{[9]}$. The gut microbiota also can change rapidly in response to environmental factors such as alterations in lifestyle, medications, or diet.

As a co-evolved system, the microbiota function almost as a "metabolic organ" that impacts nutrition and energy homeostasis. It can also be affected by, as well as contribute to, the progression of metabolic diseases, including NAFLD. In this connection, experimental evidence from animals demonstrates a direct role for gut microbiota in the development of NAFLD. Inoculation of fermentative microbial strains B. thetaiotaiomicron and M. smithii into adult germ-free (GF) C57BL/6 mice increased energy harvest from the diet as well as host adiposity regardless of reduced food intake. These microbial strains promote absorption of monosaccharides from the gut lumen, which results in induction of de novo hepatic lipogenesis ${ }^{[10]}$. Furthermore, germ-free mice were protected against Western-style diet-induced obesity ${ }^{[11]}$. A subsequent study further demonstrated that the differences in microbiota composition influenced the development of hepatosteatosis by high-fat diet (HFD) ${ }^{[12]}$. Most wild-type mice fed with HFD developed insulin resistance and systemic inflammation (responders); however, some mice remained insulin-sensitive and developed lower levels of systemic inflammation (non-responders). GF mice were then inoculated with gut microbiota from either responders or non-responders and then fed on HFD. Interestingly, nonresponder recipient mice had less hepatosteatosis and insulin resistance than responder recipient mice. Further analysis revealed that the responder mice had increased numbers of Firmicutes phylum, Barnesiella and Roseburia genera, Lachnospiraceae bacterium 609, and Barnesiella intestinihominis ${ }^{[13]}$. 


\section{DYSBIOSIS IN NAFLD PATIENTS}

Alterations in gut microbiota composition and function have negative impacts on the host (dysbiosis) and play causal roles in the development of NAFLD. In a study in which gut microbiota collected from obese donors before and after weight loss were transplanted into GF mice, the mice that received gut microbiota before weight loss had higher levels of hepatic triglyceride and cholesterol than mice that received postweight loss gut microbiota ${ }^{[14]}$. The gut microbiota in infants of obese mothers also increased inflammation and susceptibility to NAFLD ${ }^{[15]}$.

Several studies have analyzed the gut microbiota composition in NAFLD patients and control subjects ${ }^{[16]}$. Increased Proteobacteria at the phylum level was seen in NAFLD patients compared to control subjects $^{[17-21]}$. Increased Enterobacteriaceae ${ }^{[18,21]}$, decreased Rikenellaceae ${ }^{[19,22]}$ and Rumminoccaceae ${ }^{[18-21]}$ at the family level were found in NAFLD patients. Increased Escherichia ${ }^{[17,21]}$ and Dorea ${ }^{[19,23]}$ and decreased Anaerosporobacter ${ }^{[22,24]}$, Coprococcus ${ }^{[17,21,22]}$, Eubacterium ${ }^{[17,21]}$, Faecalibacterium ${ }^{[21,24]}$, and Prevotella ${ }^{[17,25]}$ were observed at the genera level in NAFLD patients. Microbial signatures of NAFLD-related fibrosis or cirrhosis also have been investigated ${ }^{[20,26-28]}$. Patients with advanced fibrosis displayed increased Gramnegative microbes and proteobacteria, and decreased Firmicutes at the phylum level. Escherichia coli and Bacteroides vulgatus were increased and Eubacterium rectale was decreased at the species level ${ }^{[20]}$. Species within the Enterobacteriaceae family ${ }^{[27]}$ and the genera Streptococcus ${ }^{[27,28]}$ and Gallibacterium ${ }^{[28]}$ were most enriched in NAFLD-cirrhosis patients. Increased Prevotella copri was associated more severe fibrosis in children with $\mathrm{NASH}^{[26]}$.

However, it is worth noting that discrepant results have been found across studies ${ }^{[16,24,29-31]}$. Variables such as exclusion criteria of subjects, ethnicity, and methods (16S-pyrosequencing vs. shotgun metagenomic sequencing) may account for these differences ${ }^{[32]}$. Nonetheless, several models that combined microbial species and a few clinical parameters were able to predict advanced fibrosis in patients with NAFLD ${ }^{[20]}$; thus, the detection of microbial markers may serve as non-invasive predictors of fibrosis [Table 1].

\section{ENDOTOXINS AND GUT MICROBIOTA METABOLITES IN NAFLD}

\section{Endotoxin effects on intestinal permeability}

The majority of the gut microbiota species are colonized in the large intestine ${ }^{[33]}$; however, clinical evidence suggests that NASH patients have a higher prevalence of small intestinal bacterial overgrowth $(\mathrm{SIBO})^{[34,35]}$. SIBO is usually defined as the presence of $>10^{5}$ colony-forming units $(\mathrm{CFU}) / \mathrm{mL}$ in duodenal aspirate cultures $^{[36]}$. The presence of SIBO in NAFLD patients is normally determined by a breath test to measure the concentration of hydrogen and/or methane in the exhaled air which is produced by intestinal bacterial metabolism ${ }^{[37]}$. Ghoshal et al ${ }^{[38]}$ used the quantitative jejunal aspirate culture and the glucose hydrogen breath test to detect low-grade SIBO in NASH patients. In this connection, Fei et al. ${ }^{[39]}$ demonstrated that three endotoxin producing strains, Enterobacter cloacae B29, Escherichia coli PY102, and Klebsiella pneumoniae A7, overgrew in the gut of two morbidly obese volunteers. When introduced to GF mice fed on HFD, E. cloacae B29 caused NAFLD, whereas HFD alone did not induce the disease. Furthermore, E. cloacae B29 induced NAFLD in a TLR4-dependent manner.

One proposed mechanism that links dysbiosis and SIBO with NAFLD is intestinal permeability and the increased circulating endotoxins levels that trigger hepatic inflammatory or fibrotic response. Intestinal permeability can be measured by lactulose/mannitol test or the urinary excretion of Cr-ethylene diamine tetraacetate ${ }^{[35,40,41]}$, and increased intestinal permeability has been demonstrated in NAFLD patients ${ }^{[2,43]}$. An animal study showed that one week of HFD feeding was able to induce enough dysbiosis to cause significant gut vascular barrier damage for bacterial translocation ${ }^{[43]}$. Several studies have also suggested that endotoxins such as Lipopolysaccharides (LPS) increased intestinal permeability through activation of 
Table 1. Changes of microbiota in NAFLD patients

\begin{tabular}{|c|c|c|c|c|}
\hline Disease & Phylum & Family & Genus & Species \\
\hline \multirow[t]{7}{*}{ NAFLD } & Proteobacteria $^{[16-20]}$ & Enterobacteriaceae $^{[17,20]}$ & Escherichia $^{[16,20]}$ & \\
\hline & & Rikenellaceae $e^{[18,21]}$ & Dorea $^{[18,22]}$ & \\
\hline & & Rumminoccaceae ${ }^{[17-20]}$ & Anaerosporobacter ${ }^{[21,23]}$ & \\
\hline & & & Coprococcus $^{[16,20,21]}$ & \\
\hline & & & Eubacterium $^{[16,20]}$ & \\
\hline & & & Faecalibacterium ${ }^{[20,23]}$ & \\
\hline & & & Prevotella ${ }^{[16,24]}$ & \\
\hline \multirow[t]{3}{*}{ Liver fibrosis or cirrhosis } & Proteobacteria ${ }^{[27]}$ & Enterobacteriaceae ${ }^{[26]}$ & Streptococcus ${ }^{[26,27]}$ & Escherichia coli[ ${ }^{[19]}$ \\
\hline & Fusobacteria $^{[27]}$ & & Gallibacterium $^{[27]}$ & Bacteroides vulgatus $^{[19]}$ \\
\hline & Bacteroidetes $^{[27]}$ & & & Eubacterium rectale ${ }^{[19]}$ \\
\hline Children with NASH & & & & Prevotella copri $^{[25]}$ \\
\hline
\end{tabular}

NAFLD: non-alcoholic fatty liver; NASH: non-alcoholic fatty liver

TLR4/MyD88 pathways ${ }^{[4,45]}$. Additionally, endotoxin levels were higher in patients with steatosis ${ }^{[46,47]}$ and were further increased after the transition from hepatosteatosis to $\mathrm{NASH}^{[41,48,49]}$.

\section{Endotoxin effects on hepatic inflammation and apoptosis}

Animal studies have also demonstrated a causative role of LPS in metabolic syndrome and hepatic steatosis. Continuous subcutaneous infusion of low-dose LPS results in increased hepatic triglyceride in mice that were fed with standard chow diet ${ }^{[50]}$. Intraperitoneal injection of LPS exacerbated liver injury in mice fed a methionine- and choline-deficient diet $(\mathrm{MCD})^{[51]}$. These studies indicated that the liver is one of the main targets of LPS, and, when the latter was bound to LPS-binding protein (LBP)-CD14 complex, it activated hepatic TLR4 to trigger the inflammatory cascade and promote NAFLD progression ${ }^{[2,53]}$. LPS also stimulates hepatocyte apoptosis, which is prominent in human $\mathrm{NASH}^{[54]}$ and may be another trigger for liver fibrosis ${ }^{[55]}$. Zhao et al ${ }^{[56]}$ demonstrated that disruption of the AMPK-caspase 6 axis caused liver damage in NASH. When AMPK signaling was impaired, caspase 6 phosphorylation was decreased and thereby activated to generate a feed-forward loop that promotes apoptosis. In mice fed MCD diet, injection of LPS intraperitoneally further increased the production of TNF $\alpha$ as well as caused cell death ${ }^{[51]}$; thus, endotoxins have effects that regulate both inflammation and cell death.

Besides endotoxins, other gut bioactive metabolites including bacterial DNA and peptidoglycans may also contribute to NAFLD progression [Figure 1]. Mitochondrial DNA strongly activated TLR9 to drive NAFLD progression ${ }^{[57-59]}$. Substituents of peptidoglycans (PGNs), such as meso-diaminopimelic acid PGN (meso-DAP PGN) and muramyl dipeptide PGN (MDP PGN), generated proinflammatory cytokines through nuclear factor- $\kappa \mathrm{B}(\mathrm{NF}-\kappa \mathrm{B}) /$ mitogen-activated protein kinase (MAPK)-dependent activation of NOD1 (Nucleotide Binding Oligomerization Domain Containing 1) and NOD2 (Nucleotide Binding Oligomerization Domain Containing 2) ${ }^{[60]}$.

\section{Altered bile acid metabolism in NASH and its effects on hepatic inflammation and apoptosis}

Bile acids are synthesized from cholesterol and in the liver. The primary bile acid species, cholic acid (CA) and chenodeoxycholic acid (CDCA), are further conjugated with glycine or taurine and stored in the gallbladder before release into the intestine after a meal ${ }^{[61]}$. In the intestine, bile acids promote the absorption of dietary fat, cholesterol, and fat-soluble vitamins. The primary bile acid species are deconjugated and dehydroxylated by intestinal microbiota to more hydrophobic secondary bile acid species, namely deoxycholic and lithocholic acid ${ }^{[62]}$, which are passively reabsorbed in the distal ileum and returned to the liver via the portal vein ${ }^{[63]}$. Some bacterial species in the colon, including Bacteroides, Clostridium, and Escherichia, can deconjugate and/or dehydroxylate bile acids, which may result in changes in the circulating unconjugated bile acids ${ }^{[64]}$. 
Normal

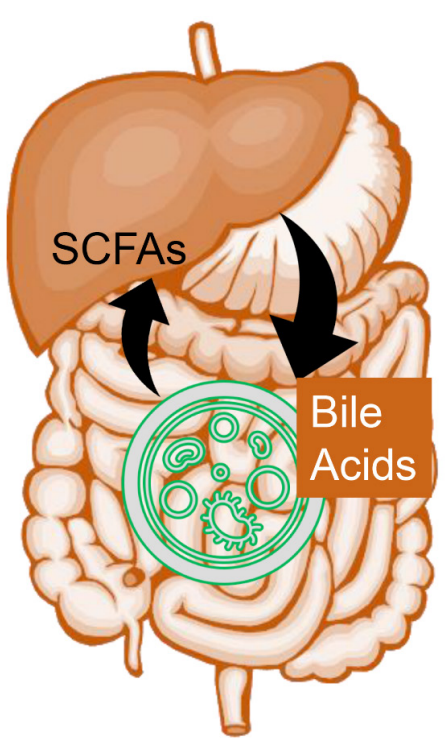

NAFLD

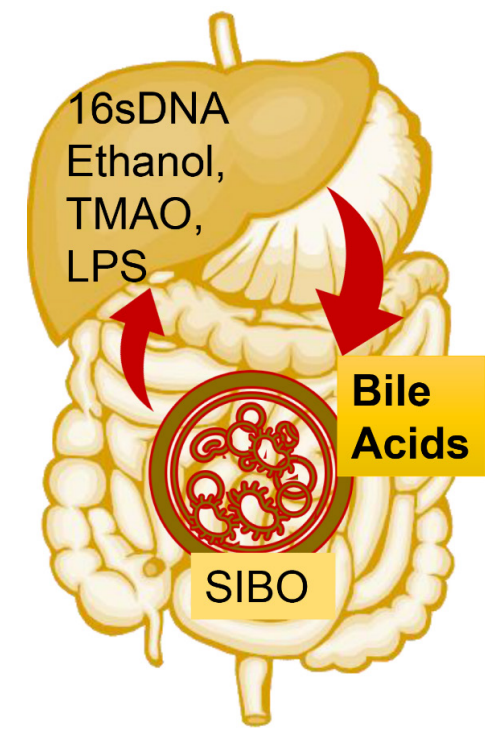

Figure 1. Gut microbial homeostatic balance is maintained under normal conditions. Gut microbiota produced SCFAs, namely acetate, butyrate, and propionate, influence hepatic metabolism by changing epigenetics/gene expression or directly via energy metabolism. Liver produced bile acids (such as cholic acids) are also processed by gut microbiota and released systemically. There is substantial microbial dysbiosis during NAFLD that causes SIBO of Gram-negative bacteria while reducing the overall microbial diversity. This bacterial overgrowth leads to produce proinflammatory molecules such as LPS, ethanol, TMAO, and bacterial 16sDNA. These proinflammatory molecules worsen the liver inflammation and fibrosis and potentially accelerate NAFLD progression. SCFAs: short chain fatty acids; SIBO: small intestinal bacterial overgrowth; LPS: Lipopolysaccharides; TMAO: trimethylamine N-oxide; NAFLD: Nonalcoholic fatty liver disease

Several studies revealed that NASH patients have higher serum levels of bile acids, including secondary bile acids, than healthy subjects ${ }^{[64,65]}$. A bile acid synthesis intermediate and marker for de novo bile acid synthesis, $7 \alpha$-hydroxy-4-cholesten-3-one (C4), was higher in the serum in NASH patients, as well as associated with changes in fecal microbiota ${ }^{[6,667}$. Some studies have suggested that there may be specific bile acid profile(s) associated with NASH. Yara et al. ${ }^{[68]}$ analyzed the serum bile acids profile of NASH patients and healthy subjects and found that ratios of primary bile acid to secondary bile acids, taurine-conjugated bile acids to glycine-conjugated bile acids, unconjugated bile acids to total bile acids, and secondary bile acids to total bile acids were decreased in NASH patients. Chen et al. ${ }^{[69]}$ showed that increased ratios of circulating conjugated chenodeoxycholic acids (CDCAs) to muricholic acids in NASH patients were positively associated with the histological severity of NASH and fibrosis. Bile acids can also function as signaling molecules to modulate various biological processes [Table 2]. Regulatory actions of bile acids are mainly mediated through its receptors, including G-protein-coupled bile acid receptor 1 (TGR5, encoded by GPBAR1 gene) and farnesoid X receptor (FXR) ${ }^{[70,71]}$. Different bile acids have variable abilities to activate these receptors ${ }^{[72]}$.

Secondary bile acids taurolithocholic acid and lithocholic acid are more potent than primary bile acids in activating TGR $5^{[73]}$. TGR5 has been found to be ubiquitously expressed in the human body with high levels of TGR5 mRNA detected in metabolically active organs such as small intestine, stomach, and liver. Activating TGR5 increased intestinal glucagon-like peptide-1 (GLP-1) release to increase glucose tolerance in obese mice ${ }^{[74]}$. TGR5 also was expressed in monocytes, macrophages, and Kupffer cells, and modulated immune response ${ }^{[73,75]}$. Indeed, in isolated Kupffer cells, bile acids activated TGR5 and inhibited LPSinduced cytokine expression in a cAMP-dependent manner ${ }^{[75]}$. 
Table 2. The effects of microbiome products on the liver

\begin{tabular}{|c|c|}
\hline Microbiome products & Effects on Liver \\
\hline Endotoxins, Bacterial DNA, Bacterial peptide glycans & $\begin{array}{l}\text { Increase gut permeability, gut inflammation, liver steatosis, inflammation, and } \\
\text { fibrosis }\end{array}$ \\
\hline Altered bile metabolites & $\begin{array}{l}\text { Increases liver bile acids conversion to unconjugated and dehydroxylated } \\
\text { secondary bile acids (e.g., deoxycholic and lithocholic acids). Activates FXR to } \\
\text { repress bile acid synthesis, regulate lipogenesis, and decrease fibrosis }\end{array}$ \\
\hline $\begin{array}{l}\text { Short-chain fatty acids (acetate. propionate, } \\
\text { butyrate) }\end{array}$ & $\begin{array}{l}\text { Stimulate gut endocrine cells to secret GLP-1 to increase hepatic fatty } \\
\text { acid } \beta \text {-oxidation. Decrease endotoxin-producing bacteria. Acetate and } \\
\text { butyrate can serve as precursors for hepatic lipogenesis; however, they } \\
\text { also can increase PPAR } \alpha \text {-mediated } \beta \text {-oxidation of fatty acids and decrease } \\
\text { hepatosteatosis }\end{array}$ \\
\hline Ethanol & $\begin{array}{l}\text { Increases gut permeability and LPS-mediated inflammation in liver. Potential } \\
\text { direct toxic effects on liver }\end{array}$ \\
\hline Choline/Choline-related metabolites & $\begin{array}{l}\text { Convert choline into TMA and then oxidized into trimethylamine } \mathrm{N} \text {-oxide } \\
\text { (TMAO) in the liver. TMAO inhibits cholesterol conversion into bile acids. } \\
\text { Associated with hepatosteatosis }\end{array}$ \\
\hline Ammonia & May increase hepatosteatosis and hepatic stellate cell activation \\
\hline
\end{tabular}

Besides their effects on inflammation, exposure to excessive bile acids in the liver can induce hepatocyte apoptosis. Bile acids activated JNK pathway and sensitized liver cells to apoptosis mediated by TNF-related apoptosis-inducing ligand (TRAIL) ${ }^{[76,77]}$. Bile acids also increased the aggregation of Fas receptor on the plasma membrane and initiated Fas receptor-mediated apoptosis ${ }^{[78,79]}$. Interestingly, bile acid-mediated apoptosis and synthesis were reduced by overexpression of an autocrine hepatic growth factor, Augmenter of Liver Regeneration (ALR) ${ }^{[80]}$.

\section{FXR effects on the liver in NASH}

FXR is highly expressed in the intestine and liver, and it can be activated by free and conjugated primary bile acids. In the small intestine, FXR activation induces the fibroblast growth factor FGF19 in human or FGF15 in mouse, which binds to FGF receptor 4 (FGFR4) in the liver to repress bile acid synthesis ${ }^{[8]]}$. Activation of hepatic FXR also regulates de novo lipogenesis, VLDL secretion, and gluconeogenesis in the liver ${ }^{[82]}$.

Synthetic FXR agonists haven been developed for treatment of NASH. Semisynthetic bile acid derivative obeticholic acid (OCA, $6 \alpha$-ethyl-chenodeoxycholic acid), previously known as INT-747, is an agonist of FXR that is 100 times more potent than CDCAs. When type 2 diabetes mellitus and NASH were treated with $25 \mathrm{mg}$ OCA for six weeks, patients had improved insulin sensitivity and decreased markers for fibrosis. These changes were associated with increased FGF19 as well as decreased levels of C4 and endogenous bile acids, consistent with FXR activation ${ }^{[83]}$. In a double-blind, placebo-controlled phase II trial for patients with NASH, when NASH patients were treated with $25 \mathrm{mg}$ OCA for 72 weeks, $45 \%$ of patients in the OCA group had improved NASH activity score and reduced hepatic fibrosis, compared with $21 \%$ of patients in the placebo group. Pruritus was observed in $23 \%$ of OCA-treated patients compared with $6 \%$ in the placebo group $^{[84]}$. In the Month 18 interim analysis of an ongoing phase III study of OCA for NASH, the histological criteria for NASH resolution endpoint was not met; however, $25 \mathrm{mg}$ OCA treatment significantly improved fibrosis and key components of NASH by other criteria among patients ${ }^{[85]}$. Additionally, OCA worsened the circulating lipid profile, as treated patients had increased low-density lipoprotein (LDL) and decreased high-density lipoprotein (HDL) cholesterol levels ${ }^{[83,84,86]}$. Similar changes were also observed when OCA was administrated to healthy human subjects ${ }^{[87]}$. However, the adverse effects on lipid profiles could be mitigated by concomitant treatment with statins ${ }^{[8 s]}$.

Several other FXR agonists have been developed to treat NASH. Cilofexor, formerly known as GS-9674 ${ }^{[89]}$, and TERN-101 (NCT04328077) have been tested in phase II clinical trials in NASH patients. Another FXR agonist, Tropifexor (TXR), is being tested in combination with cenicriviroc (CVC), a chemokine receptor types $2 / 5$ antagonist, in a phase IIb trial for NASH patients ${ }^{[90]}$. 
Furthermore, preliminary data from clinical trial also suggests that FXR activation also alters intestinal microbiota (NCT01933503). Consistent with this finding, mice fed OCA showed increased proportion of firmicutes in the small intestine ${ }^{[91]}$. Mouries et al. ${ }^{[43]}$ also demonstrate the disruption of the intestinal epithelial barrier and gut vascular barrier (GVB) are early events in NASH pathogenesis. Activation of FXR by bile acid analogue or OCA drives $\beta$-catenin activation in endothelial cells, and protect against gut vascular barrier disruption and NASH development ${ }^{[43]}$.

\section{Short-chain fatty acids}

Short-chain fatty acids (SCFAs) (acetate, propionate, and butyrate) are anaerobic fermentation products generated by gut microbiota from non-digestible carbohydrates such as non-starch polysaccharides, resistant starch, and miscellaneous low-digestible saccharides ${ }^{[92]}$. SCFAs are transferred to the liver via the portal circulation and serve as precursors for lipogenesis or gluconeogenesis ${ }^{[93]}$. There is evidence that SCFAs produced in the colon contribute to approximately $5 \%-10 \%$ of the normal daily energy requirements ${ }^{[94]}$.

Preclinical studies demonstrate that SCFAs activate the G-protein-coupled receptors (GPCRs) GPR41 and GPR43 of gut enteroendocrine L cells. Activation of GPCRs stimulates peptide YY (PYY) release, which slows gastric emptying and thus enhances nutrient absorption ${ }^{[95,96]}$. Activation of GPR41 and GPR43 in these L cells also promotes secretion of GLP-1, a peptide hormone that inhibits gastric emptying and food intake. GLP-1 also promotes hepatic lipid oxidation to reduce hepatosteatosis ${ }^{[97,98]}$. In addition, activation of GPR43 in adipocytes inhibits lipolysis and release of fatty acids into circulation ${ }^{[99]}$.

After SCFAs enter the liver via the portal vein, they undergo further metabolism. Acetate can be converted to acetyl-CoA by hepatic acetyl-coenzyme a synthetase cytoplasmic (ACSS2) ${ }^{[100]}$ and used as one of the major sources for de novo lipogenesis ${ }^{[100,101]}$. Propionate is a precursor for gluconeogenesis and promotes gluconeogenesis in the liver ${ }^{[102,103]}$. Butyrate is another precursor for hepatic de novo lipogenesis ${ }^{[102]}$. Besides functioning as metabolic substrates which feed into hepatic glucose or lipid metabolism, SCFAs can also affect hepatic metabolism by serving as signaling molecules. Propionate and butyrate activated AMPactivated protein kinase (AMPK) to increase hepatic autophagy ${ }^{[104]}$, a catabolic process which facilitates hydrolysis of triglyceride and releases free fatty acids for mitochondrial $\beta$-oxidation ${ }^{[105,106]}$. Propionate and butyrate activation of AMPK in the liver increased fatty acid oxidation and reduced HFD-induced obesity, insulin resistance, and hepatosteatosis in mice ${ }^{[107,108]}$. The activation of AMPK by SCFAs was mediated by increased UCP2 level and AMP:ATP ratio ${ }^{[104,108]}$.

SCFAs also inhibit class I and II histone deacetylases (HDACs) to modulate gene transcription. Class I and II HDACs are a group of enzymes that catalyze the removal of acetyl groups from lysine residues in histones to decrease gene transcription. Butyrate, and to a lesser degree propionate, inhibited HDACs in human colon carcinoma cells ${ }^{[109]}$. In bone marrow-derived macrophages, the inhibitory effect on HDACs by butyrate mediated its anti-inflammatory effect ${ }^{[107]}$.

Clinical studies investigated the levels of SCFAs in blood and fecal samples from patients with NAFLD. Loomba et al. ${ }^{[20]}$ demonstrated that patients with advanced fibrosis had increased levels of acetate in their fecal samples, whereas patients with mild or moderate NAFLD had increased levels of butyrate and propionate. Michail et al. ${ }^{[30]}$ found that some SCFAs, including formate, acetate, and valerate, were decreased in children with NAFLD. Moreover, when circulating SCFAs were measured in cirrhosis patients, butyric acid level inversely correlated with inflammatory markers and serum endotoxin levels ${ }^{[110]}$. The reason(s) for these apparent discrepancies may be due to differences in patient age, diagnosis, diet, environmental factors, or the manner that samples were processed and measured. With regard to the latter, the determination of actual SCFA concentrations can be problematic since they are volatile substances that 
require immediate processing for accurate measurement. These issues notwithstanding, supplementation of SCFAs in pre-clinical models of NAFLD have demonstrated beneficial effects on NAFLD. In HFD-fed mice, supplementation of butyrate decreased inflammation in the liver and adipose tissue. Furthermore, butyrate also modified bacterial population of gut microbiota by increasing SCFA-producing bacteria and decreasing endotoxin-secreting bacteria, which in turn led to increased circulating propionate and butyrate levels and decreased endotoxin levels ${ }^{[111]}$. In HFD-fed mice, butyrate increased PPAR $\alpha$-mediated $\beta$-oxidation to reduce hepatosteatosis ${ }^{[112]}$. In a fat-, fructose-, and cholesterol-rich diet, butyrate supplementation also decreased serum ALT and AST levels and attenuated the progression of NAFLD in mice ${ }^{[113]}$. Based on these preclinical data, SCFA supplementation may exert beneficial metabolic and anti-steatotic hepatic effects. Currently, there are no published studies using SCFAs to treat patients with NAFLD.

\section{Ethanol}

Low levels of endogenous ethanol can be generated by intestinal microbiota and thus may be involved in the development of NASH. This hypothesis is supported by the experimental evidence showing that $o b / o b$ mice that developed NAFLD had higher alcohol content in the morning breath than their lean littermates; moreover, this effect was abrogated by antibiotic treatment for five days ${ }^{[114]}$. Intestinal ethanol production may be due to dysbiosis since some species such as Escherichia coli can produce significant amounts of ethanol during anaerobic conditions ${ }^{[115]}$. Indeed, ethanol-producing bacteria such as Escherichia coli and other Enterobacteriaceae were substantially increased in patients with NASH ${ }^{[21]}$. However, in a human study, no significant difference was identified when the ethanol in the breath of patients with biopsyproven NASH were compared to that of healthy subjects, and only a small increase was detected in women with obesity ${ }^{[116]}$. These studies were limited because ethanol concentration in breath is an indirect way of measuring endogenous ethanol. When blood ethanol levels were actually measured, elevated circulating ethanol levels were observed in patients with NASH and were associated with upregulation of hepatic alcohol metabolic enzymes, such as alcohol, aldehyde dehydrogenases, and CYP2E ${ }^{[117]}$. Similarly, blood ethanol levels were also significantly increased in pediatric patients with $\mathrm{NASH}^{[21,118]}$. In cultured hepatocytes, ethanol showed no effect on apoptosis on its own, but it sensitized hepatocytes to TGF- $\beta$ triggered apoptosis ${ }^{[119]}$. Besides potentially toxic effects on the liver, bacteria-produced ethanol may increase intestinal permeability and portal LPS levels to activate hepatic TLR and the inflammasome cascade to contribute to liver injury ${ }^{[120]}$.

\section{Choline and choline-related metabolites}

Choline, a component of the cell membrane, is mainly obtained from red meat and eggs in the diet, although the liver can synthesize some choline endogenously ${ }^{[121]}$. In the liver, choline is required for the synthesis of very-low-density lipoprotein (VLDL). Thus, choline deficiency decreases synthesis and secretion of VLDL, leading to hepatic triglyceride accumulation ${ }^{[122,123]}$. Choline-deficient diets have been widely used in rodents to induce $\mathrm{NASH}^{[124]}$. Choline can be converted into trimethylamine (TMA) by intestinal microbiota, which can then be oxidized by hepatic monooxygenases to form trimethylamine $\mathrm{N}$-oxide (TMAO) in the liver. TMAO is then released into the circulation ${ }^{[121]}$. In HFD-fed $126 \mathrm{~S} 6$ mice, higher conversion of choline into TMA by microbiota resulted in lower bioavailability of choline ${ }^{[125]}$. TMAO may also act directly on the liver and contribute to the development of NAFLD. Gut microbiota convert dietary L-carnitine into TMAO, which reduced absorption of cholesterol in the gut lumen and the liver ${ }^{[126]}$. Dietary supplementation of TMAO also reduced bile acid synthesis enzymes and thus reduced cholesterol elimination through bile ${ }^{[126]}$. In support of these notions, clinical studies showed that serum levels of TMAO were higher in patients with NAFLD than in healthy subjects and were positively correlated with the level of steatosis ${ }^{[127]}$. Another study found that increased serum TMAO levels were significantly associated with NASH in patients with $\mathrm{T}_{2} \mathrm{DM}^{[128]}$. However, it is not known whether serum TMAO serves as a biomarker for NAFLD or other types of metabolic conditions ${ }^{[129]}$. 


\section{Ammonia}

Inability to generate urea from amino acids during end-stage liver disease, particularly during NASH and cirrhosis, can lead to hyperammonia. When severe, patients can develop hepatic encephalopathy (HE). Hyperammonia thus can be a marker to measure the severity of liver disease ${ }^{[130]}$. During NASH, ornithine transcarbamylase (OTC) and carbamoylphosphate synthetase (CPS1) mRNA, protein, and activity were reduced, leading to increased ammonia concentration ${ }^{[131]}$. Interestingly, steatosis in primary hepatic cells was also associated with OTC and CPS1 promoter hypermethylation, decreased OTC and CPS1 gene expression, and ammonia generation ${ }^{[131]}$. Moreover, ammonia itself had direct effects on hepatic stellate cells (HSCs) by activating them in cell culture and in $v i v o^{[132]}$. These findings suggested that the hyperammonemia that occurred during NASH and cirrhosis may itself contribute to the progression of fibrosis.

Ammonia also is generated from amino acids in the gut by Gram-negative anaerobes, clostridia, enterobacteria, and Bacillus spp.; Gram-positive non-sporing anaerobes, streptococci, and micrococci; and lactobacilli and yeasts that produce small amounts of ammonia ${ }^{[133]}$. Thus, the composition of the gut microbiome also contributes to circulating ammonia levels. However, the precise amount of ammonia generated by gut microbiota and their role in determining serum ammonia levels during NASH and cirrhosis is not well understood. Additionally, it is possible that endotoxin and inflammation by gut flora may contribute to increased uptake of ammonia from gut into the bloodstream and thereby contribute to the latter's toxic effect on the liver ${ }^{[132]}$.

\section{TREATMENT STRATEGY FOR NASH TARGETING GUT MICROBIOME}

The current recommended treatment for NAFLD patients is lifestyle modification, which includes, exercise, diet, and weight loss to correct of underlying risk factors such as obesity and diabetes. Pharmacologic treatments to improve insulin sensitivity, reduce oxidative stress and inflammation, or downregulate fibrosis mechanisms have been proposed for NAFLD but are not proven. There is also evidence emerging that suggests a potential role for altering gut microbiota to treat patients with NAFLD and NASH [Table 3]. Thus far, several approaches to alter the gut microbiota have been tested in NAFLD patients, including antibiotics; supplementation of pro-, pre-, or synbiotics; and fecal transplantation ${ }^{[134]}$.

\section{Antibiotics}

Several studies have examined the effect of antibiotics on NAFLD. In a preclinical model, NAFLD improved after the administration of an antibiotic cocktail (bacitracin, neomycin, and streptomycin ${ }^{[135]}$. In addition to suppressing local or systemic infections, antibiotics may also regulate inflammation caused by intestinal microbiota. In this connection, treatment with cidomycin increased small intestinal transit rate and also reduced the serum levels of ALT, AST, and TNF- $\alpha$ in NASH ${ }^{[136]}$. Gangarapu et al. ${ }^{[137]}$ also showed that rifaximin treatment significantly reduced proinflammatory cytokines, ALT, and NAFLD-liver fat score. This improvement by antibiotics was attributed to alterations in the gut microbiota population and bile acid metabolism as well as to reduced FXR signaling and decreased ceramide levels in the liver. Despite these potential beneficial effects, antibiotics need to be used judiciously and may not be appropriate therapy for most patients since they will reduce normal bacterial flora and increase the risk for overgrowth by pathogenic bacteria such as Clostridium difficile.

\section{Prebiotics, probiotics, and synbiotics}

There are numerous ongoing studies investigating the feasibility of using pre-, pro-, and synbiotics as therapeutic strategies for NAFLD/NASH. Prebiotics are indigestible food products that do not contain any living organisms but are able to promote the growth and metabolism of bacteria that ferment prebiotics to SCFAs. Probiotics are defined as viable bacteria which, upon ingestion, help improve intestinal mucosal integrity by modulating the gut microbiota to confer health benefits to the host. The combined use of preand probiotic approaches is called synbiotic (or symbiotic) therapy ${ }^{[138,139]}$. 
Table 3. Clinical trials modulating microbiome or its metabolites for NAFLD patients

\begin{tabular}{|c|c|c|c|c|}
\hline Intervention & Potential agent & NCT number & Targeted conditions & Phase \\
\hline FXR agonist & obeticholic acid & NCT02548351 & $\mathrm{NASH}$ & Phase 3 \\
\hline FXR agonist & Cilofexor & NCT03449446 & $\mathrm{NASH}$ & Phase 2 \\
\hline FXR agonist & TERN-101 & NCT04328077 & NASH & Phase 2 \\
\hline $\begin{array}{l}\text { FXR agonist in combination with } \\
\text { CCR2/5 antagonist }\end{array}$ & $\begin{array}{l}\text { Tropifexor in combination with } \\
\text { cenicriviroc }\end{array}$ & NCT03517540 & $\mathrm{NASH}$ & Phase 2 \\
\hline Synbiotic & & NCT01791959 & $\mathrm{NASH}$ & Phase 2 \\
\hline Antibiotic & Solithromycin & NCT02510599 & NASH & Phase 2 \\
\hline Probiotics & & NCT03585413 & NAFLD & Phase 3 \\
\hline FMT & & NCT02496390 & NAFLD & Phase 2 \\
\hline FMT & & NCT02970877 & NAFLD & Phase 2 \\
\hline
\end{tabular}

FXR: farnesoid X receptor; FMT: Fecal Microbiota Transplantation; NASH: non-alcoholic steatohepatitis; NAFLD: non-alcoholic fatty liver

Prebiotics might be ideal treatment candidates for NAFLD due to their low cost and safety profile; however, their effectiveness remains unproven. Prebiotic treatment of NASH patients with oligofructose $16 \mathrm{~g} / \mathrm{d}$ showed significant reduction in serum aspartate aminotransferase level; however, the same cohort had no significant difference in serum triglyceride levels when compared to placebo after eight weeks of treatment ${ }^{[140]}$. A recent meta-analysis of histologically confirmed NAFLD patients showed that use of prebiotics caused a modest reduction in serum ALT and AST but only a very small reduction in BMI. In the same cohort, no changes in serum inflammatory markers and total cholesterol were observed. In another study, prebiotics reduced low-density lipoprotein cholesterol (LDL-c) and HDL ${ }^{[141]}$. However, a systematic review of clinical studies on the use of prebiotics for obesity-induced NASH did not encourage the usage of prebiotics due to lack of quality studies ${ }^{[140]}$. Additionally, an important consideration is that different prebiotics may have distinct effects on the liver and variable effects in different individuals. Thus, the efficacy of prebiotics as a treatment for NAFLD is currently still unresolved.

Serum markers of enzymatic dysregulation or biomarkers for liver injury have been used to evaluate the efficacy of probiotics. A recent meta-analysis showed that the use of probiotics significantly reduced liver transaminase, TNF, and insulin resistance ${ }^{[142]}$. Probiotics have also been used in combination with Lactobacillus and Bifidobacteria species ${ }^{[143]}$. However, a systematic review highlighting the three clinical studies which examined the efficacy of probiotics in patients with NAFLD thus far did not support their usage in NASH due to lack of high-quality studies ${ }^{[142]}$. Nonetheless, more recent studies showed promise for this approach. Alisi et al.$^{[144]}$ showed that obese children who received probiotics composed of a mixture of eight strains daily for four months had a significantly lower risk for severe steatosis compared to placebo. Liu et al. ${ }^{[145]}$ showed that probiotic culture supernatant improved metabolic function by activating the FGF21/adiponectin pathway in a preclinical model. They showed that Lactobacillus rhamnosus culture supernatants (LGGs) reduced NASH generated by high fat/high fructose diet plus intermittent hypoxia exposure (HFDIH). The authors showed that treatment with LGGs increased hepatic FGF21 mRNA expression and circulating FGF21 protein levels, as well as increased hepatic PPAR $\alpha$ expression and fecal butyrate concentration. Further pre-clinical and clinical studies of probiotics are needed to determine their potential efficacy in $\mathrm{NAFLD}^{[145]}$.

Synbiotics have been used to treat NAFLD in animal studies and adult patients. Raso et al. ${ }^{[146]}$ showed that rodents with HFD had improvement in inflammation and decreased amounts of Enterobacteriales and Escherichia coli in colonic mucosa when given synbiotics. Malaguarnera et al. ${ }^{[147]}$ assessed 66 histologically diagnosed NASH patients receiving synbiotics for 24 weeks and found significant reduction in the TNF- $\alpha$ and C-reactive protein (CRP) levels as well as histological improvements when compared to normal control patients. In a large placebo-controlled trial $(\mathrm{N}=80)$, ultrasound-diagnosed NAFLD patients who received synbiotics for eight weeks had significant reductions in steatosis compared to their baseline levels whereas 
patients who received placebo displayed no improvement. However, no significant differences in CRP, ALT, and AST levels were observed between the two groups ${ }^{[148]}$.

When taken together, preliminary evidence suggests pre-, pro-, and synbiotic treatments for NAFLD may potentially provide clinical benefits to patients. Thus far, no severe adverse effects have been reported during any randomized control trials (RCTs), which also broadens their potential application. There are still some ongoing clinical trials (NCT03585413 and NCT01791959) examining the role of these compounds in NAFLD. Notably, the beneficial effects of pre-, pro-, and synbiotics may vary among individuals, owing to differences in dietary habits, intestinal microflora, and genetic backgrounds of the hosts. This heterogeneity introduces variability in individual responses. However, it is possible that individualizing treatments based on microbiota composition in stool and other patient considerations could improve results. Thus, optimizing the dose and determining the most effective type of therapy may be necessary for each individual.

\section{Fecal microbiota transplantation}

Fecal Microbiota Transplantation (FMT) is a new treatment approach to repopulate gut microbiota of patients with healthy intestinal flora. FMT has been successfully used in treating patients with refractory and recurrent Clostridium difficile and for other diseases by increasing the microbiome diversity ${ }^{[149,150]}$. It is an exciting new approach that is being actively explored as a treatment for NAFLD. Studies from Zhou et al. ${ }^{[151]}$ and Le Roy et al. ${ }^{[13]}$ suggested that FMT attenuated HFD-induced NASH in mice by improving intrahepatic lipid accumulation, IR, and serum proinflammatory cytokine levels. Another study showed that animals with NAFLD which underwent fecal transplant had decreased hepatic gluconeogenesis and intestinal permeability ${ }^{[152]}$. In a RCT, patients with metabolic syndrome who received gut microbiota from healthy individuals had increased insulin sensitivity and gut microbial diversity six weeks after FMT ${ }^{[153]}$. GraciaLezana et al. ${ }^{[154]}$ also showed that restoration of gut microbiota normalized portal hypertension in rodent models of NASH.

Thus far, these early studies suggest that FMT might have positive effects on NAFLD/NASH. The growing interest in FMT as a potential treatment approach for NAFLD/NASH has led to several ongoing clinical trials (NCT03803540, NCT02469272, NCT02721264, NCT02496390, and NCT02970877). However, more high quality studies are needed to determine the efficacy and safety of FMT for NAFLD. Additionally, optimization of FMT protocols, bacterial species to be transplanted, sample preparation, and dosage need to be determined.

\section{GUT MICROBIOTA AND NASH-ASSOCIATED HCC}

The transition from NAFLD to NASH involves steatosis, lobular inflammation, and progressive liver fibrosis. Although NAFLD may represent benign accumulation of liver fat alone, NASH is marked by lipotoxicity that promotes the activation of intracellular stress kinases and apoptosis ${ }^{[54]}$, leading to both inflammation and concomitant fibrosis ${ }^{[15]}$. The development of advanced NASH is worrisome clinically since some patients with this condition not only progress to cirrhosis but also have increased risk for hepatocellular carcinoma (HCC $)^{[156]}$. The first documentation of HCC associated with NASH was reported by Powell et al. ${ }^{[157]}$ in their five-year follow-up study of 42 patients with NASH. Another follow-up study showed that $2.6 \%$ of patients with NASH developed cirrhosis compared with $4 \%$ of patients with $\mathrm{HCV}^{[158]}$. A seminal study by Sanyal et al ${ }^{[159]}$ identified NAFLD/NASH as the most common underlying risk factor for HCC in the U.S., as it was present in 59\% of cases. Corroborating studies performed in Asia and Europe also have supported these findings ${ }^{[160,161]}$.

Animal models of NAFLD/NASH also show similar propensity to develop $\mathrm{HCC}^{[162]}$. Multiple hits in the form of genetic predisposition, epigenetic modifications, immunological surveillance, endocrine defects, 
and gut microbiome mediate the transition from NAFLD to $\mathrm{HCC}^{[163]}$. Hepatocyte apoptosis is also a factor that promotes $\mathrm{HCC}^{[164]}$. Detailed molecular pathogenesis of NASH-associated HCC is reviewed elsewhere ${ }^{[163,165,166]}$.

The gut microbiome plays a significant role in the progression of NASH to HCC ${ }^{[167,168]}$. NASH-associated dysbiosis and increased intestinal permeability cause the release of pathogen-associated molecular patterns (PAMPs) and gut microbiome-derived metabolites to increase liver inflammation and lipogenesis ${ }^{[23]}$. At the molecular level, PAMPs activate TLR-induced cytokine and chemokine production (e.g., IL8, IL-17, and IL1 $\beta$ ) to increase immune cell infiltration of the liver ${ }^{[169]}$. Increased cytokine surge also leads to elevated oxidative stress and DNA damage, which could then cause HCC initiation ${ }^{[170]}$. PAMPs and another microbiota-derived metabolite, lipoteichoic acid (LTA), also activate hepatic stellate cells (HSCs) via senescence-associated secretory phenotype (SASP) and promote hepatocyte proliferation and increase predisposition to $\mathrm{HCC}^{[169,171]}$.

Bile acid metabolism also plays an important role in NASH to HCC transformation. An association between altered bile acid metabolism and HCC has been documented in both human and preclinical animal studies ${ }^{[172,173]}$. High levels of bile acids in the liver can induce hepatocyte DNA damage, apoptosis, and inflammation, thus promoting tumorigenesis ${ }^{[174,175]}$. Additionally, dysbiosis in NASH leading to increased abundance of Gram-positive gut microbiota promotes HCC by augmenting the production of secondary bile acids such as deoxycholic acid (DCA), inhibiting liver sinusoidal endothelial cell (LSEC) activation and leading to chemokine ligand 6 (CXCL6) suppression, natural killer T cell recruitment, and carcinogenesis ${ }^{[176]}$. Furthermore, secondary BAs also directly contribute to HCC development by activating mTOR signaling ${ }^{[177]}$. Thus, controlling BA metabolism via antibiotics could potentially lead to prevention of HCC development ${ }^{[177]}$.

\section{CONCLUSION}

The development and progression of NAFLD is a complex and multifactorial process that involves genetic and environmental/host effects. Additionally, most NAFLD patients have co-morbidities associated with metabolic syndrome, which also impact the liver. Pre-clinical and clinical evidence suggests that altered gut microbiota, particularly the overgrowth in the small intestine and changes in the composition of microbiota, likely contribute to NAFLD progression. Although there are some general consistent changes in the gut microbiome flora, distinct gut microbiota composition can be found in different individuals, suggesting many different types of bacteria may be involved. Although gut microbiota can affect intestinal permeability, fewer than half of patients with NAFLD exhibited increased intestinal permeability, suggesting that gut metabolites or intestinal inflammation have primary effects on the liver. A few microbiota-related metabolites have been identified thus far that are positively- or negatively-associated with NAFLD progression [Table 2], although the number of identified metabolites with microbial origin currently is limited. Monitoring different metabolites as non-invasive biomarkers related to changes in the gut microbiome and NAFLD progression in stool, serum/plasma, and urine may offer the possibility for better diagnosis and personalized treatment. Understanding of the role of microbiota in NAFLD is still in its infancy. However, the identification of microbiota signatures and therapeutic modification of the microbiome provide new possibilities for the diagnosis and treatment of NAFLD.

\section{DECLARATIONS}

\section{Authors' contributions}

Conceived the manuscript: Zhou J, Yen PM

Wrote this manuscript: Zhou J, Tripathi M, Sinha RA, Singh BK

Intensive revised the manuscript: Yen PM 


\section{Availability of data and materials}

Not applicable.

\section{Financial support and sponsorship}

This work was supported by Duke-NUS Medical School and Estate of Tan Sri Khoo Teck Puat Khoo Pilot Award (Collaborative) Duke-NUS-KP (Coll)/2018/0007A to JZ, Ministry of Health, A`STAR and National Medical Research Council Singapore grants MOH-000306 (MOH-CSASI19may-0001) to PMY; MOH000319 (MOH-OFIRG19may-0002) to BKS; NMRC/OFYIRG/077/2018 to MT, and Welcome Trust/DBT India Alliance Fellowship (IA/I/16/2/502691) to RAS.

\section{Conflicts of interest}

All authors declared that there are no conflicts of interest.

\section{Ethical approval and consent to participate}

Not applicable.

\section{Consent for publication}

Not applicable.

\section{Copyright}

(c) The Author(s) 2021.

\section{REFERENCES}

1. Younossi Z, Anstee QM, Marietti M, et al. Global burden of NAFLD and NASH: trends, predictions, risk factors and prevention. Nat Rev Gastroenterol Hepatol 2018;15:11-20.

2. European Association for the Study of the L, European Association for the Study of D, European Association for the Study of O. EASLEASD-EASO Clinical Practice Guidelines for the management of non-alcoholic fatty liver disease. J Hepatol 2016;64:1388-402.

3. Eslam M, Sanyal AJ, George J, International Consensus P. MAFLD: A Consensus-Driven Proposed Nomenclature for Metabolic Associated Fatty Liver Disease. Gastroenterology 2020;158:1999-2014 e1.

4. Eslam M, Newsome PN, Sarin SK, et al. A new definition for metabolic dysfunction-associated fatty liver disease: An international expert consensus statement. $J$ Hepatol 2020;73:202-9.

5. Eslam M, Sarin SK, Wong VW, et al. The Asian Pacific Association for the Study of the Liver clinical practice guidelines for the diagnosis and management of metabolic associated fatty liver disease. Hepatol Int 2020.

6. Tilg H, Moschen AR. Evolution of inflammation in nonalcoholic fatty liver disease: the multiple parallel hits hypothesis. Hepatology 2010;52:1836-46.

7. Sender R, Fuchs S, Milo R. Are We Really Vastly Outnumbered? Revisiting the Ratio of Bacterial to Host Cells in Humans. Cell 2016;164:337-40.

8. De Filippis F, Pellegrini N, Vannini L, et al. High-level adherence to a Mediterranean diet beneficially impacts the gut microbiota and associated metabolome. Gut 2016;65:1812-21.

9. Yatsunenko T, Rey FE, Manary MJ, et al. Human gut microbiome viewed across age and geography. Nature 2012;486:222-7.

10. Backhed F, Ding H, Wang T, et al. The gut microbiota as an environmental factor that regulates fat storage. Proc Natl Acad Sci U S A 2004;101:15718-23.

11. Backhed F, Manchester JK, Semenkovich CF, Gordon JI. Mechanisms underlying the resistance to diet-induced obesity in germ-free mice. Proc Natl Acad Sci U S A 2007;104:979-84.

12. Daniel H, Gholami AM, Berry D, et al. High-fat diet alters gut microbiota physiology in mice. ISME J 2014;8:295-308.

13. Le Roy T, Llopis M, Lepage P, et al. Intestinal microbiota determines development of non-alcoholic fatty liver disease in mice. Gut 2013;62:1787-94.

14. Wang R, Li H, Yang X, et al. Genetically Obese Human Gut Microbiota Induces Liver Steatosis in Germ-Free Mice Fed on Normal Diet. Front Microbiol 2018;9:1602.

15. Soderborg TK, Clark SE, Mulligan CE, et al. The gut microbiota in infants of obese mothers increases inflammation and susceptibility to NAFLD. Nat Commun 2018;9:4462.

16. Tsai MC, Liu YY, Lin CC, et al. Gut Microbiota Dysbiosis in Patients with Biopsy-Proven Nonalcoholic Fatty Liver Disease: A CrossSectional Study in Taiwan. Nutrients 2020;12.

17. Hoyles L, Fernandez-Real JM, Federici M, et al. Molecular phenomics and metagenomics of hepatic steatosis in non-diabetic obese women. Nat Med 2018;24:1070-80. 
18. Shen F, Zheng RD, Sun XQ, Ding WJ, Wang XY, Fan JG. Gut microbiota dysbiosis in patients with non-alcoholic fatty liver disease. Hepatobiliary Pancreat Dis Int 2017;16:375-81.

19. Raman M, Ahmed I, Gillevet PM, et al. Fecal microbiome and volatile organic compound metabolome in obese humans with nonalcoholic fatty liver disease. Clin Gastroenterol Hepatol 2013;11:868-75 e1-3.

20. Loomba R, Seguritan V, Li W, et al. Gut Microbiome-Based Metagenomic Signature for Non-invasive Detection of Advanced Fibrosis in Human Nonalcoholic Fatty Liver Disease. Cell Metab 2017;25:1054-62 e5.

21. Zhu L, Baker SS, Gill C, et al. Characterization of gut microbiomes in nonalcoholic steatohepatitis (NASH) patients: a connection between endogenous alcohol and NASH. Hepatology 2013;57:601-9.

22. Wang B, Jiang X, Cao M, et al. Altered Fecal Microbiota Correlates with Liver Biochemistry in Nonobese Patients with Non-alcoholic Fatty Liver Disease. Sci Rep 2016;6:32002.

23. Del Chierico F, Nobili V, Vernocchi P, et al. Gut microbiota profiling of pediatric nonalcoholic fatty liver disease and obese patients unveiled by an integrated meta-omics-based approach. Hepatology 2017;65:451-64.

24. Wong VW, Tse CH, Lam TT, et al. Molecular characterization of the fecal microbiota in patients with nonalcoholic steatohepatitis--a longitudinal study. PLoS One 2013;8:e62885.

25. Boursier J, Mueller O, Barret M, et al. The severity of nonalcoholic fatty liver disease is associated with gut dysbiosis and shift in the metabolic function of the gut microbiota. Hepatology 2016;63:764-75.

26. Schwimmer JB, Johnson JS, Angeles JE, et al. Microbiome Signatures Associated With Steatohepatitis and Moderate to Severe Fibrosis in Children With Nonalcoholic Fatty Liver Disease. Gastroenterology 2019;157:1109-22.

27. Caussy C, Tripathi A, Humphrey G, et al. A gut microbiome signature for cirrhosis due to nonalcoholic fatty liver disease. Nat Commun 2019;10:1406.

28. Qin N, Yang F, Li A, et al. Alterations of the human gut microbiome in liver cirrhosis. Nature 2014;513:59-64.

29. Mouzaki M, Comelli EM, Arendt BM, et al. Intestinal microbiota in patients with nonalcoholic fatty liver disease. Hepatology 2013;58:120-7.

30. Michail S, Lin M, Frey MR, et al. Altered gut microbial energy and metabolism in children with non-alcoholic fatty liver disease. FEMS Microbiol Ecol 2015;91:1-9.

31. Da Silva HE, Teterina A, Comelli EM, et al. Nonalcoholic fatty liver disease is associated with dysbiosis independent of body mass index and insulin resistance. Sci Rep 2018;8:1466.

32. Aron-Wisnewsky J, Vigliotti C, Witjes J, et al. Gut microbiota and human NAFLD: disentangling microbial signatures from metabolic disorders. Nat Rev Gastroenterol Hepatol 2020;17:279-97.

33. Bures J, Cyrany J, Kohoutova D, et al. Small intestinal bacterial overgrowth syndrome. World J Gastroenterol 2010;16:2978-90

34. Wigg AJ, Roberts-Thomson IC, Dymock RB, McCarthy PJ, Grose RH, Cummins AG. The role of small intestinal bacterial overgrowth, intestinal permeability, endotoxaemia, and tumour necrosis factor alpha in the pathogenesis of non-alcoholic steatohepatitis. Gut 2001;48:206-11.

35. Miele L, Valenza V, La Torre G, et al. Increased intestinal permeability and tight junction alterations in nonalcoholic fatty liver disease. Hepatology 2009;49:1877-87.

36. Rafiei R, Bemanian M, Rafiei F, et al. Liver disease symptoms in non-alcoholic fatty liver disease and small intestinal bacterial overgrowth. Rom J Intern Med 2018;56:85-9.

37. Saad RJ, Chey WD. Breath testing for small intestinal bacterial overgrowth: maximizing test accuracy. Clin Gastroenterol Hepatol 2014;12:1964-72; quiz e119-20.

38. Ghoshal UC, Baba CS, Ghoshal U, et al. Low-grade small intestinal bacterial overgrowth is common in patients with non-alcoholic steatohepatitis on quantitative jejunal aspirate culture. Indian J Gastroenterol 2017;36:390-9.

39. Fei N, Bruneau A, Zhang X, et al. Endotoxin Producers Overgrowing in Human Gut Microbiota as the Causative Agents for Nonalcoholic Fatty Liver Disease. mBio 2020;11.

40. Volynets V, Kuper MA, Strahl S, et al. Nutrition, intestinal permeability, and blood ethanol levels are altered in patients with nonalcoholic fatty liver disease (NAFLD). Dig Dis Sci 2012;57:1932-41.

41. Giorgio V, Miele L, Principessa L, et al. Intestinal permeability is increased in children with non-alcoholic fatty liver disease, and correlates with liver disease severity. Dig Liver Dis 2014;46:556-60.

42. Luther J, Garber JJ, Khalili H, et al. Hepatic Injury in Nonalcoholic Steatohepatitis Contributes to Altered Intestinal Permeability. Cell Mol Gastroenterol Hepatol 2015;1:222-32.

43. Mouries J, Brescia P, Silvestri A, et al. Microbiota-driven gut vascular barrier disruption is a prerequisite for non-alcoholic steatohepatitis development. J Hepatol 2019;71:1216-28.

44. Nighot M, Al-Sadi R, Guo S, et al. Lipopolysaccharide-Induced Increase in Intestinal Epithelial Tight Permeability Is Mediated by TollLike Receptor 4/Myeloid Differentiation Primary Response 88 (MyD88) Activation of Myosin Light Chain Kinase Expression. Am J Pathol 2017;187:2698-710.

45. Guo S, Nighot M, Al-Sadi R, Alhmoud T, Nighot P, Ma TY. Lipopolysaccharide Regulation of Intestinal Tight Junction Permeability Is Mediated by TLR4 Signal Transduction Pathway Activation of FAK and MyD88. J Immunol 2015; 195:4999-5010.

46. Pappo I, Becovier H, Berry EM, Freund HR. Polymyxin B reduces cecal flora, TNF production and hepatic steatosis during total parenteral nutrition in the rat. J Surg Res 1991;51:106-12.

47. Pappo I, Bercovier H, Berry EM, Haviv Y, Gallily R, Freund HR. Polymyxin B reduces total parenteral nutrition-associated hepatic steatosis by its antibacterial activity and by blocking deleterious effects of lipopolysaccharide. JPEN J Parenter Enteral Nutr 
1992;16:529-32.

48. Sharifnia T, Antoun J, Verriere TG, et al. Hepatic TLR4 signaling in obese NAFLD. Am J Physiol Gastrointest Liver Physiol 2015;309:G270-8.

49. Harte AL, da Silva NF, Creely SJ, et al. Elevated endotoxin levels in non-alcoholic fatty liver disease. J Inflamm (Lond) 2010;7:15.

50. Cani PD, Amar J, Iglesias MA, et al. Metabolic endotoxemia initiates obesity and insulin resistance. Diabetes 2007;56:1761-72.

51. Kudo H, Takahara T, Yata Y, Kawai K, Zhang W, Sugiyama T. Lipopolysaccharide triggered TNF-alpha-induced hepatocyte apoptosis in a murine non-alcoholic steatohepatitis model. J Hepatol 2009;51:168-75.

52. Machado MV, Cortez-Pinto H. Gut microbiota and nonalcoholic fatty liver disease. Ann Hepatol 2012;11:440-9.

53. Abu-Shanab A, Quigley EM. The role of the gut microbiota in nonalcoholic fatty liver disease. Nat Rev Gastroenterol Hepatol 2010;7:691-701.

54. Feldstein AE, Canbay A, Angulo P, et al. Hepatocyte apoptosis and fas expression are prominent features of human nonalcoholic steatohepatitis. Gastroenterology 2003;125:437-43.

55. Canbay A, Friedman S, Gores GJ. Apoptosis: the nexus of liver injury and fibrosis. Hepatology 2004;39:273-8.

56. Zhao P, Sun X, Chaggan C, et al. An AMPK-caspase-6 axis controls liver damage in nonalcoholic steatohepatitis. Science 2020;367:652-60.

57. Garcia-Martinez I, Santoro N, Chen Y, et al. Hepatocyte mitochondrial DNA drives nonalcoholic steatohepatitis by activation of TLR9. J Clin Invest 2016;126:859-64.

58. An P, Wei LL, Zhao S, et al. Hepatocyte mitochondria-derived danger signals directly activate hepatic stellate cells and drive progression of liver fibrosis. Nat Commun 2020;11:2362.

59. Gomes MT, Campos PC, Pereira Gde S, Bartholomeu DC, Splitter G, Oliveira SC. TLR9 is required for MAPK/NF-kappaB activation but does not cooperate with TLR2 or TLR6 to induce host resistance to Brucella abortus. J Leukoc Biol 2016;99:771-80.

60. Kawasaki A, Karasudani Y, Otsuka Y, et al. Synthesis of diaminopimelic acid containing peptidoglycan fragments and tracheal cytotoxin (TCT) and investigation of their biological functions. Chemistry 2008;14:10318-30

61. Yuan L, Bambha K. Bile acid receptors and nonalcoholic fatty liver disease. World J Hepatol 2015;7:2811-8.

62. Ridlon JM, Kang DJ, Hylemon PB. Bile salt biotransformations by human intestinal bacteria. J Lipid Res 2006;47:241-59.

63. Dawson PA, Karpen SJ. Intestinal transport and metabolism of bile acids. J Lipid Res 2015;56:1085-99.

64. Ferslew BC, Xie G, Johnston CK, et al. Altered Bile Acid Metabolome in Patients with Nonalcoholic Steatohepatitis. Dig Dis Sci $2015 ; 60: 3318-28$

65. Kalhan SC, Guo L, Edmison J, et al. Plasma metabolomic profile in nonalcoholic fatty liver disease. Metabolism 2011;60:404-13.

66. Mouzaki M, Wang AY, Bandsma R, et al. Bile Acids and Dysbiosis in Non-Alcoholic Fatty Liver Disease. PLoS One 2016;11:e151829.

67. Appleby RN, Moghul I, Khan S, et al. Non-alcoholic fatty liver disease is associated with dysregulated bile acid synthesis and diarrhea: A prospective observational study. PLoS One 2019;14:e0211348.

68. Yara S IT, Miyazaki T, Murakami M, et al. Circulating bile acid profiles in Japanese patients with NASH. GastroHep 2019;1:302-10.

69. Chen J, Zheng M, Liu J, et al; Chinese NAFLD Clinical Research Network (CNAFLD CRN). Ratio of Conjugated Chenodeoxycholic to Muricholic Acids is Associated with Severity of Nonalcoholic Steatohepatitis. Obesity (Silver Spring) 2019;27:2055-66.

70. Pols TW, Noriega LG, Nomura M, Auwerx J, Schoonjans K. The bile acid membrane receptor TGR5: a valuable metabolic target. Dig Dis 2011;29:37-44.

71. Fiorucci S, Biagioli M, Zampella A, Distrutti E. Bile Acids Activated Receptors Regulate Innate Immunity. Front Immunol 2018;9:1853.

72. Molinaro A, Wahlstrom A, Marschall HU. Role of Bile Acids in Metabolic Control. Trends Endocrinol Metab 2018;29:31-41.

73. Kawamata Y, Fujii R, Hosoya M, et al. A G protein-coupled receptor responsive to bile acids. J Biol Chem 2003;278:9435-40.

74. Thomas C, Gioiello A, Noriega L, et al. TGR5-mediated bile acid sensing controls glucose homeostasis. Cell Metab 2009;10:167-77.

75. Keitel V, Donner M, Winandy S, Kubitz R, Haussinger D. Expression and function of the bile acid receptor TGR5 in Kupffer cells. Biochem Biophys Res Commun 2008;372:78-84.

76. Higuchi H, Bronk SF, Takikawa Y, et al. The bile acid glycochenodeoxycholate induces trail-receptor 2/DR5 expression and apoptosis. $J$ Biol Chem 2001;276:38610-8.

77. Higuchi H, Grambihler A, Canbay A, Bronk SF, Gores GJ. Bile acids up-regulate death receptor 5/TRAIL-receptor 2 expression via a c-Jun N-terminal kinase-dependent pathway involving Sp1. J Biol Chem 2004;279:51-60.

78. Faubion WA, Guicciardi ME, Miyoshi H, et al. Toxic bile salts induce rodent hepatocyte apoptosis via direct activation of Fas. $J$ Clin Invest 1999;103:137-45.

79. Qiao L, Studer E, Leach K, et al. Deoxycholic acid (DCA) causes ligand-independent activation of epidermal growth factor receptor (EGFR) and FAS receptor in primary hepatocytes: inhibition of EGFR/mitogen-activated protein kinase-signaling module enhances DCA-induced apoptosis. Mol Biol Cell 2001;12:2629-45.

80. Ibrahim S, Dayoub R, Krautbauer S, et al. Bile acid-induced apoptosis and bile acid synthesis are reduced by over-expression of Augmenter of Liver Regeneration (ALR) in a STAT3-dependent mechanism. Exp Cell Res 2019;374:189-97.

81. Schaap FG, Trauner M, Jansen PL. Bile acid receptors as targets for drug development. Nat Rev Gastroenterol Hepatol 2014;11:55-67.

82. Claudel T, Staels B, Kuipers F. The Farnesoid X receptor: a molecular link between bile acid and lipid and glucose metabolism. Arterioscler Thromb Vasc Biol 2005;25:2020-30.

83. Mudaliar S, Henry RR, Sanyal AJ, et al. Efficacy and safety of the farnesoid X receptor agonist obeticholic acid in patients with type 2 diabetes and nonalcoholic fatty liver disease. Gastroenterology 2013;145:574-82 e1.

84. Neuschwander-Tetri BA, Loomba R, Sanyal AJ, et al. Farnesoid X nuclear receptor ligand obeticholic acid for non-cirrhotic, nonalcoholic steatohepatitis (FLINT): a multicentre, randomised, placebo-controlled trial. Lancet 2015;385:956-65. 
85. Younossi ZM, Ratziu V, Loomba R, et al. Obeticholic acid for the treatment of non-alcoholic steatohepatitis: interim analysis from a multicentre, randomised, placebo-controlled phase 3 trial. Lancet 2019;394:2184-96.

86. Siddiqui MS, Van Natta ML, Connelly MA, et al. Impact of obeticholic acid on the lipoprotein profile in patients with non-alcoholic steatohepatitis. J Hepatol 2020;72:25-33.

87. Pencek R, Marmon T, Roth JD, Liberman A, Hooshmand-Rad R, Young MA. Effects of obeticholic acid on lipoprotein metabolism in healthy volunteers. Diabetes Obes Metab 2016;18:936-40.

88. Pockros PJ, Fuchs M, Freilich B, et al. CONTROL: A randomized phase 2 study of obeticholic acid and atorvastatin on lipoproteins in nonalcoholic steatohepatitis patients. Liver Int 2019;39:2082-93.

89. Patel K, Harrison SA, Elkhashab M, et al. Cilofexor, a Nonsteroidal FXR Agonist, in Patients With Noncirrhotic NASH: A Phase 2 Randomized Controlled Trial. Hepatology 2020;72:58-71.

90. Pedrosa M, Seyedkazemi S, Francque S, et al. A randomized, double-blind, multicenter, phase $2 \mathrm{~b}$ study to evaluate the safety and efficacy of a combination of tropifexor and cenicriviroc in patients with nonalcoholic steatohepatitis and liver fibrosis: Study design of the TANDEM trial. Contemp Clin Trials 2020;88:105889.

91. Friedman ES, Li Y, Shen TD, et al. FXR-Dependent Modulation of the Human Small Intestinal Microbiome by the Bile Acid Derivative Obeticholic Acid. Gastroenterology 2018;155:1741-52 e5.

92. Yajima M, Karaki SI, Tsuruta T, et al. Diversity of the intestinal microbiota differently affects non-neuronal and atropine-sensitive ileal contractile responses to short-chain fatty acids in mice. Biomed Res 2016;37:319-28.

93. Wolever TM, Brighenti F, Royall D, Jenkins AL, Jenkins DJ. Effect of rectal infusion of short chain fatty acids in human subjects. Am J Gastroenterol 1989;84:1027-33.

94. McNeil NI. The contribution of the large intestine to energy supplies in man. Am J Clin Nutr 1984;39:338-42.

95. Samuel BS, Shaito A, Motoike T, et al. Effects of the gut microbiota on host adiposity are modulated by the short-chain fatty-acid binding G protein-coupled receptor, Gpr41. Proc Natl Acad Sci U S A 2008;105:16767-72.

96. Musso G, Gambino R, Cassader M. Obesity, diabetes, and gut microbiota: the hygiene hypothesis expanded? Diabetes Care 2010;33:2277-84.

97. Svegliati-Baroni G, Saccomanno S, Rychlicki C, et al. Glucagon-like peptide-1 receptor activation stimulates hepatic lipid oxidation and restores hepatic signalling alteration induced by a high-fat diet in nonalcoholic steatohepatitis. Liver Int 2011;31:1285-97.

98. Tolhurst G, Heffron H, Lam YS, et al. Short-chain fatty acids stimulate glucagon-like peptide-1 secretion via the G-protein-coupled receptor FFAR2. Diabetes 2012;61:364-71.

99. Ge H, Li X, Weiszmann J, et al. Activation of G protein-coupled receptor 43 in adipocytes leads to inhibition of lipolysis and suppression of plasma free fatty acids. Endocrinology 2008;149:4519-26.

100. Zhao S, Jang C, Liu J, et al. Dietary fructose feeds hepatic lipogenesis via microbiota-derived acetate. Nature 2020;579:586-91.

101. Hanson RW, Ballard FJ. The relative significance of acetate and glucose as precursors for lipid synthesis in liver and adipose tissue from ruminants. Biochem $J$ 1967;105:529-36.

102. den Besten G, Lange K, Havinga R, et al. Gut-derived short-chain fatty acids are vividly assimilated into host carbohydrates and lipids. Am J Physiol Gastrointest Liver Physiol 2013;305:G900-10.

103. Weidemann MJ, Hems R, Williams DL, Spray GH, Krebs HA. Gluconeogenesis from propionate in kidney and liver of the vitamin B12deficient rat. Biochem J 1970;117:177-81.

104. Iannucci LF, Sun J, Singh BK, et al. Short chain fatty acids induce UCP2-mediated autophagy in hepatic cells. Biochem Biophys Res Commun 2016;480:461-7.

105. Singh R, Kaushik S, Wang Y, et al. Autophagy regulates lipid metabolism. Nature 2009;458:1131-5.

106. Sinha RA, You SH, Zhou J, et al. Thyroid hormone stimulates hepatic lipid catabolism via activation of autophagy. J Clin Invest 2012;122:2428-38.

107. Gao Z, Yin J, Zhang J, et al. Butyrate improves insulin sensitivity and increases energy expenditure in mice. Diabetes 2009;58:1509-17.

108. den Besten G, Bleeker A, Gerding A, et al. Short-Chain Fatty Acids Protect Against High-Fat Diet-Induced Obesity via a PPARgammaDependent Switch From Lipogenesis to Fat Oxidation. Diabetes 2015;64:2398-408.

109. Waldecker M, Kautenburger T, Daumann H, Busch C, Schrenk D. Inhibition of histone-deacetylase activity by short-chain fatty acids and some polyphenol metabolites formed in the colon. J Nutr Biochem 2008;19:587-93.

110. Juanola O, Ferrusquia-Acosta J, Garcia-Villalba R, et al. Circulating levels of butyrate are inversely related to portal hypertension, endotoxemia, and systemic inflammation in patients with cirrhosis. FASEB J 2019;33:11595-605.

111. Zhai S, Qin S, Li L, Zhu L, Zou Z, Wang L. Dietary butyrate suppresses inflammation through modulating gut microbiota in high-fat dietfed mice. FEMS Microbiol Lett 2019;366.

112. Sun B, Jia Y, Hong J, et al. Sodium Butyrate Ameliorates High-Fat-Diet-Induced Non-alcoholic Fatty Liver Disease through Peroxisome Proliferator-Activated Receptor alpha-Mediated Activation of beta Oxidation and Suppression of Inflammation. J Agric Food Chem 2018;66:7633-42.

113. Baumann A, Jin CJ, Brandt A, et al. Oral Supplementation of Sodium Butyrate Attenuates the Progression of Non-Alcoholic Steatohepatitis. Nutrients 2020;12.

114. Cope K, Risby T, Diehl AM. Increased gastrointestinal ethanol production in obese mice: implications for fatty liver disease pathogenesis. Gastroenterology 2000;119:1340-7.

115. Dawes EA, Foster SM. The formation of ethanol in Escherichia coli. Biochim Biophys Acta 1956;22:253-65.

116. Nair S, Cope K, Risby TH, Diehl AM. Obesity and female gender increase breath ethanol concentration: potential implications for the 
pathogenesis of nonalcoholic steatohepatitis. Am J Gastroenterol 2001;96:1200-4.

117. Baker SS, Baker RD, Liu W, Nowak NJ, Zhu L. Role of alcohol metabolism in non-alcoholic steatohepatitis. PLoS One 2010;5:e9570.

118. Engstler AJ, Aumiller T, Degen C, et al. Insulin resistance alters hepatic ethanol metabolism: studies in mice and children with nonalcoholic fatty liver disease. Gut 2016;65:1564-71.

119. Gaitantzi H, Meyer C, Rakoczy P, et al. Ethanol sensitizes hepatocytes for TGF-beta-triggered apoptosis. Cell Death Dis 2018;9:51.

120. Parlesak A, Schafer C, Schutz T, Bode JC, Bode C. Increased intestinal permeability to macromolecules and endotoxemia in patients with chronic alcohol abuse in different stages of alcohol-induced liver disease. $J$ Hepatol 2000;32:742-7.

121. Wang Z, Klipfell E, Bennett BJ, et al. Gut flora metabolism of phosphatidylcholine promotes cardiovascular disease. Nature 2011;472:57-63.

122. Mehedint MG, Zeisel SH. Choline's role in maintaining liver function: new evidence for epigenetic mechanisms. Curr Opin Clin Nutr Metab Care 2013;16:339-45.

123. Yao ZM, Vance DE. Reduction in VLDL, but not HDL, in plasma of rats deficient in choline. Biochem Cell Biol 1990;68:552-8.

124. Stephenson K, Kennedy L, Hargrove L, et al. Updates on Dietary Models of Nonalcoholic Fatty Liver Disease: Current Studies and Insights. Gene Expr 2018;18:5-17.

125. Dumas ME, Barton RH, Toye A, et al. Metabolic profiling reveals a contribution of gut microbiota to fatty liver phenotype in insulinresistant mice. Proc Natl Acad Sci U S A 2006;103:12511-6.

126. Koeth RA, Wang Z, Levison BS, et al. Intestinal microbiota metabolism of L-carnitine, a nutrient in red meat, promotes atherosclerosis. Nat Med 2013;19:576-85.

127. Chen YM, Liu Y, Zhou RF, et al. Associations of gut-flora-dependent metabolite trimethylamine-N-oxide, betaine and choline with nonalcoholic fatty liver disease in adults. Sci Rep 2016;6:19076.

128. Leon-Mimila P, Villamil-Ramirez H, Li XS, et al. Trimethylamine N-oxide levels are associated with NASH in obese subjects with type 2 diabetes. Diabetes Metab 202010.1016/j.diabet.2020.07.010.

129. Barrea L, Annunziata G, Muscogiuri G, et al. Trimethylamine-N-oxide (TMAO) as Novel Potential Biomarker of Early Predictors of Metabolic Syndrome. Nutrients 2018;10.

130. De Chiara F, Thomsen KL, Habtesion A, et al. Ammonia Scavenging Prevents Progression of Fibrosis in Experimental Nonalcoholic Fatty Liver Disease. Hepatology 2020;71:874-92.

131. De Chiara F, Heeboll S, Marrone G, et al. Urea cycle dysregulation in non-alcoholic fatty liver disease. J Hepatol 2018;69:905-15.

132. Thomsen KL, De Chiara F, Rombouts K, et al. Ammonia: A novel target for the treatment of non-alcoholic steatohepatitis. Med Hypotheses 2018;113:91-7.

133. Richardson AJ, McKain N, Wallace RJ. Ammonia production by human faecal bacteria, and the enumeration, isolation and characterization of bacteria capable of growth on peptides and amino acids. BMC Microbiol 2013;13:6.

134. Wiest R, Albillos A, Trauner M, Bajaj JS, Jalan R. Targeting the gut-liver axis in liver disease. J Hepatol 2017;67:1084-103.

135. Jiang C, Xie C, Li F, et al. Intestinal farnesoid X receptor signaling promotes nonalcoholic fatty liver disease. J Clin Invest 2015;125:386402.

136. Wu WC, Zhao W, Li S. Small intestinal bacteria overgrowth decreases small intestinal motility in the NASH rats. World J Gastroenterol 2008;14:313-7.

137. Gangarapu V, Ince AT, Baysal B, et al. Efficacy of rifaximin on circulating endotoxins and cytokines in patients with nonalcoholic fatty liver disease. Eur J Gastroenterol Hepatol 2015;27:840-5.

138. Paolella G, Mandato C, Pierri L, Poeta M, Di Stasi M, Vajro P. Gut-liver axis and probiotics: their role in non-alcoholic fatty liver disease. World J Gastroenterol 2014;20:15518-31.

139. Hu H, Lin A, Kong M, et al. Intestinal microbiome and NAFLD: molecular insights and therapeutic perspectives. J Gastroenterol 2020;55:142-58.

140. Jayakumar S, Loomba R. Review article: emerging role of the gut microbiome in the progression of nonalcoholic fatty liver disease and potential therapeutic implications. Aliment Pharmacol Ther 2019;50:144-58.

141. Perdomo CM, Fruhbeck G, Escalada J. Impact of Nutritional Changes on Nonalcoholic Fatty Liver Disease. Nutrients 2019;11.

142. Xiao MW, Lin SX, Shen ZH, Luo WW, Wang XY. Systematic Review with Meta-Analysis: The Effects of Probiotics in Nonalcoholic Fatty Liver Disease. Gastroenterol Res Pract 2019;2019:1484598.

143. Ma YY, Li L, Yu CH, Shen Z, Chen LH, Li YM. Effects of probiotics on nonalcoholic fatty liver disease: a meta-analysis. World $J$ Gastroenterol 2013;19:6911-8.

144. Alisi A, Carpino G, Nobili V. Paediatric nonalcoholic fatty liver disease. Curr Opin Gastroenterol 2013;29:279-84.

145. Liu Q, Liu Y, Li F, et al. Probiotic culture supernatant improves metabolic function through FGF21-adiponectin pathway in mice. $J$ Nutr Biochem 2020;75:108256.

146. Raso GM, Simeoli R, Iacono A, et al. Effects of a Lactobacillus paracasei B21060 based synbiotic on steatosis, insulin signaling and tolllike receptor expression in rats fed a high-fat diet. J Nutr Biochem 2014;25:81-90.

147. Malaguarnera G, Leggio F, Vacante M, et al. Probiotics in the gastrointestinal diseases of the elderly. J Nutr Health Aging 2012;16:402-10.

148. Asgharian A, Askari G, Esmailzade A, Feizi A, Mohammadi V. The Effect of Symbiotic Supplementation on Liver Enzymes, C-reactive Protein and Ultrasound Findings in Patients with Non-alcoholic Fatty Liver Disease: A Clinical Trial. Int J Prev Med 2016;7:59.

149. Song Y, Garg S, Girotra M, et al. Microbiota dynamics in patients treated with fecal microbiota transplantation for recurrent Clostridium difficile infection. PLoS One 2013;8:e81330.

150. Kelly CR, Khoruts A, Staley C, et al. Effect of Fecal Microbiota Transplantation on Recurrence in Multiply Recurrent Clostridium difficile Infection: A Randomized Trial. Ann Intern Med 2016;165:609-16. 
151. Zhou D, Pan Q, Shen F, et al. Total fecal microbiota transplantation alleviates high-fat diet-induced steatohepatitis in mice via beneficial regulation of gut microbiota. Sci Rep 2017;7:1529.

152. Porras D, Nistal E, Martinez-Florez S, Gonzalez-Gallego J, Garcia-Mediavilla MV, Sanchez-Campos S. Intestinal Microbiota Modulation in Obesity-Related Non-alcoholic Fatty Liver Disease. Front Physiol 2018;9:1813.

153. Vrieze A, Van Nood E, Holleman F, et al. Transfer of intestinal microbiota from lean donors increases insulin sensitivity in individuals with metabolic syndrome. Gastroenterology 2012;143:913-6 e7.

154. Garcia-Lezana T, Raurell I, Bravo M, et al. Restoration of a healthy intestinal microbiota normalizes portal hypertension in a rat model of nonalcoholic steatohepatitis. Hepatology 2018;67:1485-98.

155. Day CP, Saksena S. Non-alcoholic steatohepatitis: definitions and pathogenesis. J Gastroenterol Hepatol 2002;17 Suppl 3:S377-84.

156. Hardy T, Oakley F, Anstee QM, Day CP. Nonalcoholic Fatty Liver Disease: Pathogenesis and Disease Spectrum. Annu Rev Pathol 2016;11:451-96.

157. Powell EE, Cooksley WG, Hanson R, Searle J, Halliday JW, Powell LW. The natural history of nonalcoholic steatohepatitis: a follow-up study of forty-two patients for up to 21 years. Hepatology 1990;11:74-80.

158. Ascha MS, Hanouneh IA, Lopez R, Tamimi TA, Feldstein AF, Zein NN. The incidence and risk factors of hepatocellular carcinoma in patients with nonalcoholic steatohepatitis. Hepatology 2010;51:1972-8.

159. Sanyal A, Poklepovic A, Moyneur E, Barghout V. Population-based risk factors and resource utilization for HCC: US perspective. Curr Med Res Opin 2010;26:2183-91.

160. Kim GA, Lee HC, Choe J, et al. Association between non-alcoholic fatty liver disease and cancer incidence rate. J Hepatol 201710.1016/ j.jhep.2017.09.012.

161. Pais R, Fartoux L, Goumard C, et al. Temporal trends, clinical patterns and outcomes of NAFLD-related HCC in patients undergoing liver resection over a 20-year period. Aliment Pharmacol Ther 2017;46:856-63.

162. Tsuchida T, Lee YA, Fujiwara N, et al. A simple diet- and chemical-induced murine NASH model with rapid progression of steatohepatitis, fibrosis and liver cancer. J Hepatol 2018;69:385-95.

163. Raza S, Rajak S, Anjum B, Sinha RA. Molecular links between non-alcoholic fatty liver disease and hepatocellular carcinoma. Hepatoma Res 2019;5:42.

164. Hirsova P, Bohm F, Dohnalkova E, et al. Hepatocyte apoptosis is tumor promoting in murine nonalcoholic steatohepatitis. Cell Death Dis 2020;11:80

165. Michelotti GA, Machado MV, Diehl AM. NAFLD, NASH and liver cancer. Nat Rev Gastroenterol Hepatol 2013;10:656-65.

166. Cholankeril G, Patel R, Khurana S, Satapathy SK. Hepatocellular carcinoma in non-alcoholic steatohepatitis: Current knowledge and implications for management. World J Hepatol 2017;9:533-43.

167. Ponziani FR, Bhoori S, Castelli C, et al. Hepatocellular Carcinoma Is Associated With Gut Microbiota Profile and Inflammation in Nonalcoholic Fatty Liver Disease. Hepatology 2019;69:107-20.

168. Chen YH, Wu WK, Wu MS. Microbiota-Associated Therapy for Non-Alcoholic Steatohepatitis-Induced Liver Cancer: A Review. Int $J$ Mol Sci 2020;21.

169. Dapito DH, Mencin A, Gwak GY, et al. Promotion of hepatocellular carcinoma by the intestinal microbiota and TLR4. Cancer Cell 2012;21:504-16

170. Yuan D, Huang S, Berger E, et al. Kupffer Cell-Derived Tnf Triggers Cholangiocellular Tumorigenesis through JNK due to Chronic Mitochondrial Dysfunction and ROS. Cancer Cell 2017;31:771-89 e6.

171. Loo TM, Kamachi F, Watanabe Y, et al. Gut Microbiota Promotes Obesity-Associated Liver Cancer through PGE2-Mediated Suppression of Antitumor Immunity. Cancer Discov 2017;7:522-38.

172. Wang X, Fu X, Van Ness C, Meng Z, Ma X, Huang W. Bile Acid Receptors and Liver Cancer. Curr Pathobiol Rep 2013;1:29-35.

173. Chen T, Xie G, Wang X, et al. Serum and urine metabolite profiling reveals potential biomarkers of human hepatocellular carcinoma. Mol Cell Proteomics 2011;10:M110 004945.

174. Jansen PL. Endogenous bile acids as carcinogens. J Hepatol 2007;47:434-5.

175. Knisely AS, Strautnieks SS, Meier Y, et al. Hepatocellular carcinoma in ten children under five years of age with bile salt export pump deficiency. Hepatology 2006;44:478-86.

176. Ma C, Han M, Heinrich B, et al. Gut microbiome-mediated bile acid metabolism regulates liver cancer via NKT cells. Science 2018;360.

177. Yamada S, Takashina Y, Watanabe M, et al. Bile acid metabolism regulated by the gut microbiota promotes non-alcoholic steatohepatitisassociated hepatocellular carcinoma in mice. Oncotarget 2018;9:9925-39. 